\title{
Together at last: crystallography and NMR
}

In a collaborative high-throughput effort to determine the three-dimensional structures of small proteins, two studies demonstrate that X-ray crystallography and nuclear magnetic resonance (NMR) spectroscopy are complementary techniques.

The sequencing of entire genomes is certainly an incredible feat, yet the really hard work has only begun. The current challenge in the post-genomic era is to identify the structure and function of all the encoded protein products. Even using the high-throughput approach known as structural proteomics, elucidating the three-dimensional structures of all naturally occurring proteins is likely to be a physically impossible task. But some brave researchers, such as the members of the Northeast Structural Genomics Consortium (NESG), are attempting to make some progress as part of the larger Protein Structure Initiative, with the specific objective of obtaining a structure for at least one member of every large protein family.

NMR spectroscopy has gained acceptance in the last few decades as a powerful technology for protein structure determination, but it is limited to small $(<40 \mathrm{kDa})$, stable, soluble proteins that do not aggregate at the high concentrations required for data collection. Because of these limitations, many researchers have been skeptical about the potential impact of NMR in structural proteomics, as the popular view has remained that virtually any protein that can be studied by NMR can eventually be coaxed to yield a crystal for X-ray crystallography. But Cheryl Arrowsmith of the University of Toronto, Gaetano Montelione of Rutgers University and their NESG collaborators hope to change that belief, demonstrating in tandem papers that NMR and crystallography can have complementary roles in structural proteomics (Snyder et al., 2005; Yee et al. 2005).

Time, of course, is a central consideration in a structural proteomics approach. Typically, NMR suitability screening proceeds quickly, but data collection and processing can take months. Conversely, hundreds of crystallization conditions must be screened, often followed by further optimization (and accompanied by lots of patience), but once a diffraction-quality crystal is obtained, data collection and processing can be done in a single day. By subjecting all small proteins in their structural pipeline to both two-dimensional ${ }^{15} \mathrm{~N}-{ }^{1} \mathrm{H}$ heteronuclear single-quantum coherence (HSQC) correlation spectroscopy and large-scale screening of crystallization conditions, Arrowsmith and Montelione optimize their chances for successful structure solution. "We feel very strongly that both methodologies are partially overlapping but not completely, and that you will get your structural information more quickly and efficiently if you use both," says Arrowsmith.

Notably, through separate studies totaling 422 proteins, Arrowsmith and Montelione both discovered that a significant number of proteins were amenable only to NMR. Montelione explains: "There was one platform for producing proteins, crystallization and NMR screening in Toronto, and a parallel separate effort at Rutgers using different constructs, different molecular biology, different protocols, yet we ended up with the same result at the end of the day." They also found statistical evidence contradicting the widely held belief that the quality of the HSQC spectrum correlates with the probability of obtaining suitable crystals. "Some proteins will give nice spectra but did not provide crystals, and some proteins that gave poor spectra did yield crystal structures," says Montelione.

Arrowsmith and Montelione hope their work will demonstrate that NMR is an important tool for structural proteomics, as new technologies continue to make data collection and analysis speedier. "Almost half of the NESG structures have come from NMR," reports Arrowsmith. "We're one of the few structural proteomics groups that puts strong emphasis on both NMR and crystallography, so we're in a good position to compare the two methodologies."

\section{Allison Doerr}

\section{RESEARCH PAPERS}

Snyder, D.A. et al. Comparisons of NMR spectral quality and success in crystallization demonstrate that NMR and X-ray crystallography are complementary methods for small protein structure determination. J. Am. Chem. Soc. 127, 16505-16511 (2005).

Yee, A.A. et al. NMR and X-ray crystallography, complementary tools in structural proteomics of small proteins. J. Am. Chem. Soc. 127, 16512-16517 (2005). 